\title{
Successful long-term ambulatory norepinephrine infusions in a patient with pure autonomic failure
}

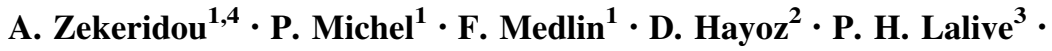 \\ T. Kuntzer ${ }^{1}$
}

Received: 8 January 2015 / Accepted: 18 February 2015/Published online: 3 July 2015

(C) Springer-Verlag Berlin Heidelberg 2015

\begin{abstract}
We present a case study of a patient with pure autonomic failure who was successfully treated with ambulatory norepinephrine (NE) infusions over a 9-year-period of time before death occurred unexpectedly. Given this patient's response to the NE infusion treatment, we discuss the option of ambulatory NE infusions as a treatment for severe orthostatic hypotension that is refractory to common treatments.
\end{abstract}

Keywords PAF . Pure autonomic failure .

Norepinephrine infusions

\section{Introduction}

Pure autonomic failure (PAF), or Bradbury-Eggleston syndrome, is a degenerative disorder of the peripheral autonomic nervous system mainly characterized by progressive, disabling orthostatic hypotension $(\mathrm{OH})$. Other signs of

Drs. A. Zekeridou and P. Michel contributed equally to this article as joint first authors.

\footnotetext{
A. Zekeridou

Anastasia.zek@gmail.com

1 Nerve-Muscle Unit, Department of Clinical Neurosciences, Lausanne University Hospital CHUV, Lausanne, Switzerland

2 Department of Internal Medicine, Fribourg University Hospital, Fribourg, Switzerland

3 Laboratory Medicine Service, Division of Neurology, Department of Clinical Neurosciences, Department of Genetic Medicine and Laboratory, Geneva University Hospital HUG, Geneva, Switzerland

4 Present Address: Laboratory of Neuroimmunology, Mayo Clinic College of Medicine, Rochester, MN 55905, USA
}

autonomic dysfunction may also be present, such as gastrointestinal and urinary symptoms, sudomotor disturbances, or impotence [1]. As do all patients with $\mathrm{OH}, \mathrm{PAF}$ patients have an impaired quality of life with episodes of syncope and pre-syncope that often leave them confined to a bed or to a chair. We present the case of a PAF patient in whom ambulatory norepinephrine (NE) infusions were used successfully.

\section{Case report}

A previously healthy 48 -year-old man first drew attention to his symptoms in 1999 by reporting a 2-year-duration of sexual dysfunction with loss of erection, along with decreased physical endurance. Progressively, he developed an increase in his daily need to sleep and presented several episodes of syncope in relation to exertion. On neurological examination, an isolated $\mathrm{OH}$ could be demonstrated repeatedly after $1 \mathrm{~min}$ standing, with a drop of $>60 \mathrm{mmHg}$ of his systolic blood pressure (BP) and a drop of $>30 \mathrm{mmHg}$ of his diastolic BP, with the heart rate (HR) being unaltered. Nerve conduction studies of the upper and lower extremities were normal. The sympathetic skin response was absent in his soles; the $\mathrm{R}-\mathrm{R}$ heart variation rate with a breathing frequency of $6 / \mathrm{min}$ was reduced $(1.03$, normal $>2.25$ ). During a tilt-table test, continuous arterial BP and HR monitoring (Finapress ${ }^{\circledR}$ ) confirmed syncope (Fig. 1a).

A large workup was performed (and later partially repeated every year) and found no cause for this autonomic dysfunction. More specifically, there was no evidence of amyloid deposition (biopsies of abdominal fat, peroneus brevis muscle, and sural nerve were normal) or of autoimmune $\mathrm{OH}$ (there was an absence of serum antibodies 
Fig. 1 Tilt test with blood pressure (BP) and heart rate (HR) recorded by continuous non-invasive measurements (Finapress ${ }^{\circledR}$ ), without (a) and with (b) the norepinephrine (NE) pump infusion at a rate of $160-320 \mathrm{ng} / \mathrm{kg}$ body weight/ min. b BP and HR are totally dependent on the exogenous NE infusion
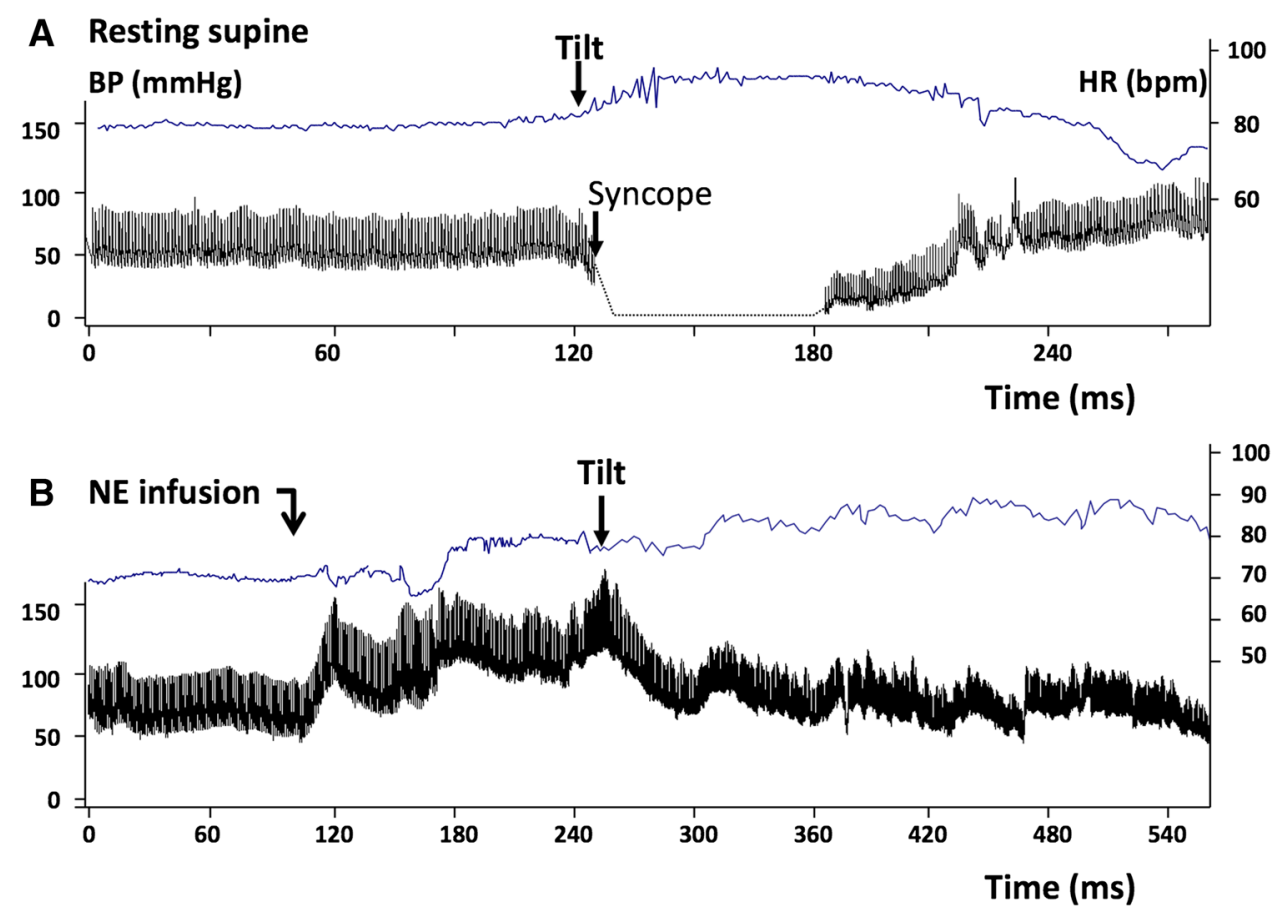

against the ganglionic nicotinic acetylcholine receptor). The ${ }^{123}$ I-metaiodobenzylguanidine (MIBG) myocardial scintigraphy showed no tracer uptake, indicating loss of post-ganglionic sympathetic innervation; the $\mathrm{NE}$ and vasopressin blood measurements in the horizontal and upright positions showed an abnormally excessive increase in vasopressin measures during the upright position $(>20 \mathrm{pg} / \mathrm{ml}$, normal $<2)$ and low values of $\mathrm{NE}$ in the supine $(<100 \mathrm{pg} /$ $\mathrm{ml}$, normal $>250)$ and upright $(<100 \mathrm{pg} / \mathrm{ml}$, normal $>400)$ positions; both findings suggestive of PAF [2, 3].

The patient was initially treated with non-pharmacological measures (fluid and salt intake, elastic stockings, meal fragmentation), followed by pharmacological treatments (midodrine up to $40 \mathrm{mg} /$ day and fludrocortisone up to $0.8 \mathrm{mg} / \mathrm{day}$ ) for 24 months with benefit. However, in 2001 he presented with progressive worsening of his condition, he was not able to stand up, and eventually he was unable to leave the home. At this time a NE infusion with a portable pump was proposed in the literature and we decided to use it for this patient, after explaining the advantages and the possible risks of that procedure and obtaining his consent during an interdisciplinary meeting [4].

During a 2-week hospital stay in the neurological intermediate care unit NE requirements were determined in the supine, sitting, and upright positions using continuous non-invasive monitoring of vital signs. Then, a permanent left subclavian venous bloodline was implanted and connected to a portable medication pump (CADD-Legacy PLUS $^{\circledR}$, Deltec Inc., St. Paul, MN). With the activation of the pump 30-60 s before changing position from supine to sitting or standing, syncope disappeared; the improvement was also demonstrated by a tilt-table test (Fig. 1b). About $60 \mathrm{~s}$ before lying down, the patient would stop the pump. There was no drug delivery during the night while the patient was in bed. The patient would restart the pump 30-60 s before standing up. Once the patient had learned and used this regimen for 3 days in the hospital setting without complications, he was discharged and continued to use this regimen independently, with regular blood pressure monitoring as described above. No particular adjustment was made for regular daily activities, but the patient avoided strenuous exercise such as jogging because of a perceived lack of endurance. The patient started working again part-time and was able to stop all other medication. Because of slowly increasing blood pressure drops in the upright position on self-monitoring, with associated presyncopal symptoms, the pump infusion rate needed to be adjusted only twice in the 9-year follow-up (from an infusion rate of 160 to $320 \mathrm{ng} / \mathrm{kg}$ body weight $/ \mathrm{min}$ in the standing position, corresponding to $5 \mathrm{ml} / \mathrm{h}$ of solution containing $15 \mathrm{mg} / 100 \mathrm{ml}$ of $\mathrm{NE}$ ) and the patient continued to have an almost normal life in between; however, as expected, activities were limited by loss of endurance. The effect of a catecholamine spillover on skeletal muscle membrane excitability could be demonstrated just after the administration of NE [5], but the patient reported persistent fatigue and fatigability that did not improve by using amantadine or modafinil. Annual cardiac echocardiography demonstrated a moderate progressive decrease in ejection fraction from an initially normal value to $50 \%$ at the last 
recording. There were no segmental wall abnormalities suggestive of ischemia, but neither a cardiac stress test nor coronarography was performed. No infection of the infusion system was observed; an expert nurse for pump systems used in diabetic patients repeatedly supervised and instructed the patient regarding proper use and hygienic measures. Two episodes of obstruction of the system were reported in the first year and required rinsing of the catheter with anticoagulants. After 9 years of treatment, the patient died unexpectedly during his sleep without any previous cardiovascular symptoms. No autopsy was performed. It was hypothesized that this could have been due to cardiotoxicity, acute coronary syndrome, or cardiac arrhythmia from prolonged NE treatment, but arrhythmia related to the underlying disease may also be a potential cause of death.

\section{Discussion}

Our treated patient underscores the typical findings and outcome in PAF, and illustrates successful chronic treatment of PAF by parenteral infusion of NE; it is also probable that the use of $\mathrm{NE}$ infusions was associated with his death.

Progressive $\mathrm{OH}$ is the heralding manifestation of several disorders. The neurological workup aims to recognize amyloid, infectious, or diabetic neuropathies, inflammatory or paraneoplastic diseases, or other neurodegenerative disorders [1]. In our case, MIBG scintigraphy and the NE and vasopressin measurements indicated peripheral autonomic nervous dysfunction suggestive of PAF, after exclusion of other causes. The patient had presented with supine nocturnal hypertension previously requiring antihypertensive medication; its resolution with $\mathrm{NE}$ infusion therapy in the upright position is likely due to the decreased need for salt retention during the day, which had led to nocturnal (supine) hypertension with the patient's previous pharmacological regimen. After losing the initial beneficial effects of midodrine and fludrocortisone treatment in this patient, we immediately proposed the NE pump infusions given the severity of the symptoms; other drugs such as subcutaneous octreotide, yohimbine, droxidopa, or domperidone may also be considered before proceeding to invasive management as in our patient [1]. This report confirms the results of Oldenburg's study describing six patients successfully treated with the NE pump infusions in which none of them suffered complications due to arterial hypo- or hypertension, or infection of the infusion system [4]. Multi-disciplinary (neurologist/nephrologist/internist/ pharmacologist), expert evaluation, neuro-hormonal assessment data, and blood pressure monitoring are helpful in coping with PAF and assessing the best treatment options, including NE constant infusions. To our knowledge, this is the first description of a patient with such a long lasting and efficient NE treatment for PAF. Given that the unexpected death of our patient may be related to the NE treatment (cardiac arrest due to cardiotoxicity, acute coronary syndrome cardiac arrhythmia from prolonged NE treatment, or arrhythmia related to the underlying disease), this treatment option should be considered as a possibility only in severe autonomic failure, while utilizing multidisciplinary collaborations and frequent follow-ups, and always taking into consideration the possibility of adverse events.

Conflict of interest On behalf of all authors, the corresponding author states that there is no conflict of interest.

\section{References}

1. Benarroch EE (2014) The clinical approach to autonomic failure in neurological disorders. Nat Rev Neurol 10:396-407

2. Kaufmann H, Oribe E, Miller M, Knott P, Wiltshire-Clement M, Yahr M (1992) Hypotension-induced vasopressin release distinguishes between pure autonomic failure and multiple system atrophy with autonomic failure. Neurology 42:590-593

3. Kashihara K, Ohno M, Kawada S, Okumura Y (2006) Reduced cardiac uptake and enhanced washout of ${ }^{123} \mathrm{I}-\mathrm{MIBG}$ in pure autonomic failure occurs conjointly with Parkinson's disease and dementia with Lewy bodies. J Nucl Med 47:1099-1102

4. Oldenburg O, Mitchell A, Nu J, Koeppen S (2001) Ambulatory norepinephrine treatment of severe autonomic orthostatic hypotension. J Am Coll Cardiol 37:219-223

5. Kuntzer T, Michel P (2004) Muscle membrane polarisation after provocative tests, and after cooling: the normal CMAP changes to be expected. Clin Neurophysiol 115:1457-1463 